\title{
Unabhängige Justiz - Gegengewicht zur Erosion von Grundrechten in Europa $^{*}$
}

Dass es ein europäisches Kriminaljustizsystem gibt, ist nicht mehr die Frage. Aber eine Antwort, wie es beschaffen sein soll, gibt es auch nicht. Das Europarecht war und ist in seiner Tendenz auf die Integration von Wirtschafts- und Sozialsystemen angelegt, denen der physisch unmittelbare Zwang des Strafrechts - der immer noch im hoheitlich und öffentlich veranlassten Entzug der Freiheit seinen Ausdruck findet - wesensfremd ist. Folglich eignen sich vorhandene Legitimationsprogramme europäischer Rechtsentwicklung nicht unmittelbar für das europäische Kriminaljustizsystem. Wir sind derzeit mit dem Problem konfrontiert, wie und ob die vorhandene Programmierung des Europarechts auf Systemintegration auch auf das Kriminaljustizsystem übertragbar ist. Davon betroffen ist die Judikative, die sich als europäische, unabhängige und unparteiliche Straf-Gewalt fortentwickeln muss. Abgekoppelt vom Nationalstaat traditionellen Zuschnitts unterliegt sie neuen Anforderungen: legitimatorischen (I), inhaltlichen (II) und organisatorischen (III).

\section{I. „Wesen ohne Seele“? Die Funktion der Europäischen Justiz zwischen System- und politischer Integration}

Was und in welchem Umfang darf die Judikative kontrollieren? Das Gesetz, seine Anwendung, sein Inhalt, seine Genese selbst? Montesquieu sieht die Gefahr des Richters als einem potentiellen „Unterdrücker“, wenn seine Funktion nicht klar von Exekutive und Legislative getrennt ist. ${ }^{1}$ Daraus leitet sich der berühmte Satz von den „Richtern der Nation“ ab, die lediglich der „Mund“ seien, der den „Wortlaut des Gesetzes spreche“, „Wesen ohne Seele gleichsam, die weder die Stärke noch die Strenge des Gesetzes mäßigen können". ${ }^{2}$ Diese Funktionsbestimmung steht in Zusammenhang mit einem Gesetzesbegriff der Aufklärung, der das Gesetz als die Verkörperung höherer praktischer Vernunft verstand. Gesetzes- und Freiheitsbegriff gehören zusammen. Stimmen die Gesetze mit dem Freiheitsbegriff überein, so geben sie zugleich auch die Grenzen der eigenen Freiheit, des individuellen Dürfens an. Die Anwendung und der Erhalt dieses „Geists der Gesetze“ macht das Organisationsprinzip strikter Gewaltenteilung als

* Dieser Einleitungsbeitrag ist zugleich ein Editorial für einige Beiträge der Tagung Criminal Justice in Europe: Challenges, Principles and Perspectives in Luxembourg, vom 22.-23. Oktober 2010.

1 Montesquieu, Vom Geist der Gesetze, 1965, S. 213.

2 Montesquieu, aaO., S. 221. 
eines Prinzips der Freiheit selbst notwendig, die als ,,politische Freiheit“ praktisch wirksam werden soll. ${ }^{3}$

Unparteilich gegenüber gesellschaftlicher Macht und unabhängig von deren Einfluss ist die Justiz daher auf die Anwendung des ,richtigen“ Gesetzes beschränkt. Eine darüber hinaus gehende Kontrollfunktion gegenüber dem Gesetzgeber, ja gar die Intervention in Legislativgewalt selbst kommt ihr nicht $\mathrm{zu}$. Indes verliert der vernunftimprägnierte Gesetzesbegriff an Strahlkraft. Das Rechtssystem erbringt eine Steuerungsleistung für die Gesellschaft. Dabei soll es nicht mehr nur auf individuellen Rechtsschutz ankommen, sondern auf die Vermittlung von Wohlstand und Sicherheit. Recht ist eine Ausfallbürgschaft für die Risikogesellschaft. Die Bindung an das Gesetz ist nur noch lose. In der Folge lockert sich auch die Bindung des Richters, des Verfassungsrichters insbesondere, an den Gesetzgeber. Es eröffnet sich der Justiz ein Raum moralischer Grundsatz- und politischer Zielsetzungserwägungen. Eine Prämisse des Präventionsstaates bestand auch in einem materiellen Rechtsbegriff, der es der Judikative ermöglichte, den demokratischen Gesetzgebungsprozess mit vermeintlich objektiven Wertordnungen zu unterlegen. Die Justiz tritt so an die Stelle des Gesetzgebers, erweitert ihren Entscheidungsspielraum auf Kosten des Demokratieprinzips. ${ }^{4}$ Ein über Abwehrfunktion hinausgehendes Verständnis von Grundrechten als einer objektiven, Staat und Gesellschaft insgesamt durchdringenden Ordnung riskiert dabei, an die Justizgeschichte der Weimarer Republik anzuknüpfen, als die Dritte Gewalt durchaus in Anwendung materieller Rechtsbegriffe die eigene politische, auf Restauration gerichtete Macht gegenüber dem demokratischen Gesetzgeber durchsetzen wollte und durchgesetzt hat.

Trotz dieser historischen Erfahrung gibt es keine unmittelbare Rückkehr zu einem liberalen und funktionsbegrenzten Verständnis von Justiz als Hüterin von Grund- und Freiheitsrechten, wenn man sie nur kontextabhängig von einer funktionierenden liberalen Wirtschaftsgesellschaft interpretiert. ${ }^{5}$ Diesen scheinbaren Kontext überwindend, will die Diskurstheorie die justizielle Kontrolle von Grundrechten über ihre Abwehrfunktion hinaus erweitern und sie im Rahmen des modernen Demokratieprinzips justieren. Nach ihrer Lesart haben demokratische Verfahren die Vermutung praktischer Vernunft ${ }^{6}$ für sich, wenn sie die Staatsbürger in die Lage versetzen, in Wahrnehmung ihres Selbstbestimmungsrechts und in wechselseitiger Anerkennung ihrer Partizipationsrechte kooperativ das politische Projekt gerechter Lebensverhältnisse zu verfolgen. ${ }^{7}$ Justizielle Kontrolle erstreckt sich demnach auf die „Verfahrensbedingungen der demokratischen Genese“ von Gesetzen und schützt nicht einen spezifischen Inhalt von

3 Montesquieu, aaO, XI. Buch, Drittes Kapitel, ,Worin die Freiheit besteht“. „(...) Jedoch bedeutet politische Freiheit nicht, da $\beta$ man machen kann, was man will. In einem Staat, das heißt einer mit Gesetzen ausgestatteten Gesellschaft, kann Freiheit lediglich bedeuten, da $\beta$ man $\mathrm{zu}$ tun vermag, was man wollen soll, und man nicht zu tun gezwungen wird, was man nicht wollen soll (...)“.

4 Die Diskussion ist geführt bei P.-A. Albrecht, Das Strafrecht auf dem Weg vom liberalen Rechtsstaat zum sozialen Interventionsstaat, in: ders.: Der Weg in die Sicherheitsgesellschaft. Auf der Suche nach staatskritischen Absolutheitsregeln, 2010, S. 259 ff. Vgl. auch Denninger, Das Bundesverfassungsgericht zwischen Recht und Politik, in: ders., Das Recht in globaler Unordnung, 2005, S. $96 \mathrm{ff}$.

5 Vgl. Habermas, Faktizität und Geltung, S. 328 f.

6 Habermas, Faktizität und Geltung, S. 333.

7 Vgl. Habermas, Faktizität und Geltung, S. 320. 
Grundrechten, sondern die Formen, die die Ausübung privater und öffentlicher Autonomie von Staatsbürgern erst ermöglichen. ${ }^{8}$ Die Legitimation von Recht und Staat entspringt aus den Formen kommunikativer, öffentlich verantworteter und deliberativer Politik. Das Recht seinerseits gewinnt seine Legitimität nicht als eine äußere Ordnung, die der Politik auferlegt wird, sondern als ein System, das demokratieverstärkend politische Kooperation unter freien und gleichen Staatsbürgern absichert. Folglich kann verfassungsrechtliche Kontrolle soweit in den Gesetzgebungsprozess intervenieren, als seine prozeduralen Voraussetzungen missachtet werden. ${ }^{9}$ Eine auf einen ,idealisierten“ richtigen Inhalt gemünzte Kontrolle indes findet nicht statt. ${ }^{10}$

Was aber geschieht, wenn ein solch prozedurales Paradigma auf einen Gegenstand trifft, der noch ungeformt stetem Wandel unterworfen ist? Könnte es sein, dass die diskurstheoretische Sicht auf die Funktion justizieller Kontrolle auf den gefestigten nationalen Verfassungsstaat angewiesen bleibt? Im europäischen Rechtsraum klaffen Lücken einer Legitimation durch Verfahren. Das Bundesverfassungsgericht hat in seinem Urteil zum Vertrag von Lissabon ${ }^{11}$ diese Lücken soweit geschlossen als es die enge Kopplung des europäischen Gesetzgebungsprozesses an nationales Verfassungsrecht sicherstellte. Das betrifft insbesondere das Strafrecht, das - als Bestandteil des Raums der Freiheit, der Sicherheit und des Rechts - erhöhten prozeduralen Anforderungen genügen muss und daher die wirksame Beteiligung der nationalen Parlamente notwendige Bedingung europäischer Strafgesetzgebung ist. ${ }^{12}$ Die Durchsetzung demokratischer Verfahrensbedingungen mittels justizieller Kontrolle funktioniert so immer mit dem Bezug auf das etablierte Verfassungsrecht des Nationalstaats.

Damit ist aber noch nichts über die Funktion justizieller Kontrolle innerhalb eines sich verselbständigenden europäischen Kriminaljustizsystems gesagt. Der Rechtsbegriff ist auf der Befugnis zum Zwang begründet. ${ }^{13}$ Die Legitimation des Strafrechts hängt nicht nur prozedural, sondern auch materiell davon ab, unter welchen Voraussetzungen, nach welchen Bedingungen und mit welchen Folgen gestraft werden darf. Als typische Zwangsordnung bedarf es auf europäischer Ebene spezifischer Kriterien, die der Legitimation des Strafrechts zu eigen sind. Strafrechtliche Prinzipien wie Gesetzlichkeit, Schuld oder faires Verfahren aber gilt es im europäischen Systemzusammenhang des Strafrechts erst noch fortzuentwickeln. Hier gibt es einerseits zum Teil noch zu wenig justizielle Kontrolle, was die Garantie dieser Prinzpien anbelangt, und andererseits zum Teil bereits zu viel justizielle Kontrolle, was den Ausbau der Strafgesetzgebung durch Annexkompetenz betrifft. Ein prozedurales Paradigma allein kann den Schutz privater und öffentlicher Autonomie europäischer Bürger gegenüber und innerhalb der Gesetzgebung nicht verbürgen. Das Strafrecht zeigt, dass es nicht nur um Teil-

8 Vgl. Habermas, aaO.

9 Habermas, aaO., S. 340.

10 Habermas, aaO.

11 BVerfG, 2 BvE 2/08 vom 30.6.2009.

12 BVerfG, aaO., Abs. 363 und 365.

13 Kant, Metaphysik der Sitten, Ausgabe der Preussischen Akademie der Wissenschaften Band VI, S. 231. An der Stelle heisst es genauer: ,(...), wenn ein gewisser Gebrauch der Freiheit selbst ein Hindernis der Freiheit nach allgemeinen Gesetzen (d.i. unrecht) ist, so ist der Zwang, der diesem entgegengesetz wird, als Verhinderung eines Hindernisses der Freiheit mit Freiheit nach allgemeinen Gesetzen zusammen stimmend, d.i. recht (...)“. 
habe geht, sondern um den Schutz vor Zwang, der sich nicht nur durch die Teilnahme an der Entscheidung über Zwangsgesetze erschöpft. Die europäische (Straf-)Justiz fungiert als Siegelwahrerin von Freiheitsrechten, die dem politischen Gestaltungsprozess vorgelagert sind, die eingeklagt und verbindlich implementiert werden müssen.

In Europa haben Einzelfälle, an denen sich der Schutz von Grundrechten erproben muss, stets eine über den Fallbezug hinausgehende Bedeutung. Gestärkte Grundrechte im Einzelfall können den europäischen Gesetzgeber verpflichten, die politischen Bemühungen um universelle Verfahrensgarantien in einem europäischen Kriminaljustizsystem zu verstärken. Die Justiz darf kein Ersatzgesetzgeber sein. Ihr geht es nicht um den Ausbau justizieller Macht um ihrer selbst willen. Sie muss aber um so stärker als Hüterin von Freiheits- und Grundrechten auftreten und ihre Kontrollfunktion um so offensiver interpretieren, je lückenhafter das Verfahren demokratischer Gesetzgebung ist und je ungesicherter individuelle Rechtspositionen sind.

Noch immer ist die Europäische Union durch die Antinomie von Systemintegration und politischer Integration geprägt. ${ }^{14}$ Die Steuerung des Binnenmarktes und die Absicherung der vier Grundfreiheiten unterliegen partiell einer eigenen Logik, die von Normen, Prinzipien, Werten und der Verständigung darüber abkoppelt. ${ }^{15}$ Politische Integration in Europa findet dagegen nur langsam statt und bleibt oft auf den Nationalstaat begrenzt. ${ }^{16}$ Der Europäische Gerichtshof hat seine Funktion bislang überwiegend als Beitrag zur Systemintegration im Sinne einer Verrechtlichung von Wirtschaft und Verwaltung auf europäischer Ebene begriffen. Mit dem Vertrag von Lissabon werden Grundrechte zum verbindlichen Element jeglicher europäischen Rechtsetzung, sei es in Anwendung der EU-Grundrechtecharta, sei es als Teil verfassungsrechtlicher Prinzipien der Mitgliedstaaten. Der mögliche Beitritt der EU zur Europäischen Menschenrechtskonvention verstärkt den Rechtsrahmen der politischen Integration Europas, die sich nicht nur ökonomisch, sondern als öffentlicher Raum freier und gleicher Unionsbürger versteht. Künftig wird also justizielle Kontrolle in Europa - ausgeübt durch gesamteuropäische Gerichte - ihren Beitrag nicht nur zur Systemintegration, sondern auch zur politischen Integration, deren Grammatik sich erst durch die Implementation gesamteuropäisch wirksamer Grundrechte ergibt, leisten müssen. Das europäische Kriminaljustizsystem wird zum Seismographen, inwiefern diese politische Integration gelingt, oder nicht.

\section{Europäische Rechtsprechung zwischen nationaler Souvernänität und europäischer Rechtskontrolle}

Noch bleibt die europäische Justiz in ihrer Funktion, eine Grammatik des Rechts für die politische Integration Europas mitzuentwerfen, ambivalent. Sie belässt einerseits dem nationalen demokratischen Gesetzgeber einen weiten Ermessens- und Gestaltungsspielraum bei der konkreten Ausgestaltung des Grundrechtsschutzes, auch dann, wenn er

14 Vgl. Habermas, Staatsbürgerschaft und nationale Identität, in : Faktizität und Geltung, S. $632 \mathrm{ff}$.

15 Vgl. Habermas, aaO., S. 644.

16 Habermas, aaO., S. 645. 
europäisches Recht umsetzt. In „Advocaaten voor de Wereld“17 wandte der Europäische Gerichtshof den Maßstab des Gesetzlichkeitsprinzips erst gar nicht auf die in Art. 2 Abs. 2 des Rahmenbeschlusses zum Europäischen Haftbefeh ${ }^{18}$ aufgelisteten Straftatentypen an. Nach dem EuGH soll es lediglich darauf ankommen, ob die Straftat, derentwegen der Haftbefehl im Ausstellungsmitgliedstaat erlassen wurde, den Kriterien der Gesetzesbestimmtheit genügt. ${ }^{19}$ In seiner „Tillack-Entscheidung ${ }^{\text {“20 }}$ verneinte der EuGH die rechtliche Verbindlichkeit von Art. 10 VO 1073/99, der einen Informationstransfer der Europäischen Betrugsermittlungsbehörde OLAF an die Staatsanwaltschaften der Mitgliedstaaten umfasst. Er entzog damit alle auf der Grundlage von Art. 10 VO 1073/99 erfolgten Ermittlungshandlungen grundrechtlicher Überprüfung. Schließlich verzichtet das Urteil zur Speicherung von Vorratsdaten ${ }^{21}$ auf die Anwendung datenzschutzrechtlicher Grenzen, da die Speicherung selbst nicht als Akt der Strafverfolgung begriffen wird. So unterschiedlich die Gegenstände der Entscheidungen sind, so eint sie doch ein Grundproblem europäischen Rechtsschutzes, das sich vor allem in Strafrecht auswirkt. Stets geht es um Eingriffe in Grundrechtspositionen, die zwar unmittelbar im nationalen Recht Wirkung entfalten, die aber durch europäisches Recht veranlasst sind. Der mittelbare Rechtsgrund eines Grundrechtseingriffs aber entzieht sich so einer europäischen Justizkontrolle, die über Kompetenzfragen hinausweist. Das System der Strafgesetzgebung differenziert sich vertikal und horizontal aus. Justizielle Kontrolle folgt dieser Ausdifferenzierung nicht immer. Daraus erst erwächst das Problem lückenhaften oder in seiner Reichweite sehr variablen Schutzes strafrechtlicher Prinzipien.

Andererseits beginnt sich die justizielle Kontrolle durch europäische Gerichte zunehmend auf Bereiche ursprünglich nationaler Souveränität zu erstrecken, die vor einiger Zeit dem europäischen Zugriff noch verschlossen gewesen wären. Sowohl nationale Gesetzgebung als auch Exekutive werden erst im europäischen Kontext auf die Einhaltung von Grundrechten verpflichtet. In seinem Urteil „Salduz gegen Türkei“"22 hat der Europäische Gerichtshof für Menschenrechte das Recht auf einen Verteidiger ab dem frühest möglichen Zeitpunkt eines beginnenden Strafverfahrens - jedenfalls ab dem Zeitpunkt polizeilicher Vernehmung - statuiert. ${ }^{23}$ Der Zugang zu einem Anwalt sei wesentlicher Bestandteil des nemo-tenetur-Prinzips. ${ }^{24}$ Einschränkungen seien nur dann zulässig, wenn die Garantie des fairen Verfahrens insgesamt nicht darunter leide. ${ }^{25}$ Eingriffe in das Recht können jedenfalls dann nicht gerechtfertigt werden, wenn der Entzug des Verteidigers dazu führt, dass Beschuldigtenrechte schon zu einem frühen Zeitpunkt

17 Europäischer Gerichtshof, Urteil vom 3. Mai 2007, Rs C-303/05.

18 2002/584/JI : Rahmenbeschluss des Rates vom 13. Juni 2002 über den Europäischen Haftbefehl und die Übergabeverfahren zwischen den Mitgliedstaaten (...), Abl. L 190 vom 18. Juli 2002, $1 \mathrm{ff}$.

19 EuGH, Rs. C-303/05, Rz. 53 f.

20 EuGH, Rs. T-193/04, Beschluss vom 15. Oktober 2004 und EuGH Rs. C-521/04, Beschluss vom 19. April 2005.

21 Urteil des Gerichtshofs vom 10. Februar 2009, Rs. C-301/06; Richtlinie 2006/24 des Europäischen Parlaments und des Rates vom 15. März 2006.

22 Salduz v. Turkey, application no. 36391/02, Urteil vom 27. November 2008.

23 Salduz v. Turkey, Para. 52.

24 Salduz v. Turkey, Para. 54.

25 Salduz v. Turkey, Para. 51. 
des Verfahrens - etwa einer ersten Vernehmung - irreparabel beschädigt werden. ${ }^{26}$ Für einige der Unterzeichnerstaaten zog die Rechtsprechung des EGMR die rechtliche Notwendigkeit nach sich, ihr Strafverfahrensrecht zu reformieren. ${ }^{27}$ Für die Europäische Kommission ergab sich aus dem Urteil der kriminalpolitische Mehrwert, offensiver die schrittweise Verwirklichung gesamteuropäischer Verfahrensgarantien vorantreiben zu können. ${ }^{28}$ Deren kriminalpolitische Initiative, das Recht auf einen Anwalt als europäisches Verfahrensrecht zu verankern, ist untrennbar mit dem „Salduz-Urteil“ verknüpft. ${ }^{29}$

Im Fall „S und Marper gegen Vereinigtes Königreich“ ${ }^{“ 30}$ hat der EGMR den Ermessensspielraum der Unterzeichnerstaaten bei der Reichweite des Datenschutzes eingeschränkt und europaweit verbindliche Kriterien zum Schutz der Privatsphäre im Sinne von Art. 8 EMRK entfaltet. Danach stellt die unbestimmte Speicherung von Vorratsdaten unverdächtiger oder nicht verurteilter Personen einen ungerechtfertigten Eingriff in Art. 8 EMRK dar. ${ }^{31}$ Eine solche Speicherung erhöht das Risiko der Stigmatisierung und ist insofern mit der Unschuldsvermutung nicht zu vereinbaren. ${ }^{32}$ Kein öffentliches Interesse, auch nicht in Fragen der inneren Sicherheit, kann das private Rechtsschutzinteresse Unschuldiger an der Unversehrtheit ihrer Privatsphäre überwiegen. ${ }^{33}$ Auch dieses Urteil steckte den Rechtsrahmen für künftige Eingriffe in das Recht auf informationelle Selbstbestimmung ab und muss bei der künftigen europäischen Neuregelung der Speicherung von Vorratsdaten maßgebend sein.

Nicht nur die Straßburger, sondern auch die Luxemburger Richter haben die justizielle Kontrolle von Grundrechten dort verstärkt, wo der durch den europäischen Gesetzgeber verabschiedete Gesetzestext lückenhaft und die durch die europäische Exekutive praktizierte Anwendung grundrechtsgefährdend ist. So wird im Eurostat-Fall ${ }^{34}$ deutlich: Bei der europäischen Betrugsermittlung müssen Verteidigungsrechte schon im administrativen Vorverfahren berücksichtigt werden. Im Falle einer Verletzung können die dadurch erlangten Beweise unverwertbar sein. Im Unterschied zur Tillack-Entscheidung stellen sich Ermittlungsmaßnahmen der europäischen Betrugsbekämpfungsbehörde (OLAF) stets als solche als justiziell überprüfbar dar, unabhängig davon, ob sie sich unmittelbar auf strafprozessuale oder disziplinarische Maßnahmen in den Mitgliedstaaten selbst auswirken. ${ }^{35}$ In seinen Urteilen zum Einfrieren von Vermögen aufgrund des

26 Salduz v. Turkey, Para. 54.

27 Vgl. Europäische Kommission, Generaldirektion Justiz, Reflection Paper for Experts' meeting, Brussels 11 and 12 October 2010, S. $6-8$.

28 Vgl. etwa Resolution of the Council of 30 November 2009 on a Roadmap for strengthening procedural rights of suspected or accused persons in criminal proceedings, Abl. C 295/1 vom 4.12.2009.

29 Vgl. auch Europäische Kommission, $\operatorname{COM}(2010) 392$ final, Proposal for a Directive of the European Parliament and of the Council on the right to information in criminal proceedings, Brussels 20.7.2010.

30 EGMR, Application nos. 30562/04 und 30566/04, Urteil vom 4. Dezember 2008.

31 EGMR, aaO., Para. 66.

32 EGMR, aaO., Para. 122.

33 EGMR, aaO., Para. 127.

34 EuG T-48/05, Urteil vom 8.7.2008.

35 Vgl. näher Braum, JZ 2009, S. 298 ff. 
Verdachts, Mitglied einer terroristischen Vereinigung zu sein, ${ }^{36}$ hat der Europäische Gerichtshof klargestellt, dass europäische Grundrechte bei jeder Maßnahme mit unmittelbarer Wirkung auf einen Unionsbürger - unabhängig auf welche Rechtsquelle die Maßnahme gestützt wird - unverbrüchlich Geltung beanspruchen. Man sieht, dass sich die Ausdifferenzierung des europäischen Kriminaljustizsystems in der europäischen Rechtsprechung in Strafsachen widerspiegeln kann. In vertikaler Hinsicht wird nationales Recht in einen verbindlichen internationalen europäischen Rechtsrahmen gestellt, der seinerseits auf den nationalen Gesetzgeber zurückwirkt. Horizontal - auf europäischer Ebene selbst - sieht sich die Europäische Union, wenn sie ihre Kompetenzen in Strafsachen ausübt, einer stärker kontrollierenden europäischen Judikative gegenüber, die den europäischen Grundrechtsschutz verbindlich und kontinuierlich fortentwickelt. Dem Risiko einer weiteren Erosion von Grundrechten, die durch die Europäisierung des Strafrechts veranlasst wird, stehen Anzeichen eines durch die europäische Justiz ausgelösten Verrechtlichungsschubs gegenüber.

\section{Europäische Rechtsprechung zwischen Binnenmarktromantik und Strafrecht}

Der Europäische Gerichtshof ist seit der Gründung der Europäischen Gemeinschaften auch das Gericht des Europäischen Binnenmarktes. Seine Rechtsprechung wird als Motor europäischer Integration verstanden. Wo die Politik zögerte, wies der EuGH den Weg. Die vier Grundfreiheiten des Binnenmarktes, die Rechte von Marktteilnehmern und Verbrauchern sind in europäischer Rechtsprechung etabliert und konsequent an Einzelfällen weiterentwickelt worden. Der europäische Binnenmarkt und sein Rechtsrahmen sind treffliches Beispiel für die Systemintegration durch Recht. Nur die Zeiten ändern sich. Einstmals mögen die Europäische Gemeinschaften durch ihre wirtschaftlichen Probleme, durch Butterberge und Milchseen, charakterisiert worden sein, standen Probleme des grenzüberschreitenden Handels und der gegenseitigen Anerkennung von Produktbezeichnungen im Vordergrund, war fraglich, was Schnaps, was Bier sein darf. Nun aber wird es ernst: Das Problem, wer und nach welchen Kriterien den mit einer Freiheitsstrafe oftmals verbundenen sozialen Tod erleidet, weist über bloße Systemdefizite hinaus. Letztere mögen gar gegenüber dem harten Entzug der Freiheit eines Bürgers fast romantisch anmuten. Ist der Europäische Gerichtshof organisatorisch aber bereit, diese neue Realität europäischen Rechts als solche zu akzeptieren und sie normativ überzeugend zu bewältigen?

Anfänge sind zumindest gemacht. Der europäische Haftbefehl, der sich bei aller berechtigten Kritik zugleich auch als Motor europäischer Strafrechtspflege entpuppen könnte, zog auch die Justizreform eines beschleunigten Vorabentscheidungsverfahrens nach sich. ${ }^{37}$ Unionsbürger, die in einem Mitgliedstaat in Untersuchungshaft sitzen, müssen - soll das Verfahren fair sein - in angemessener Verfahrensdauer Klarheit über die Rechtslage erhalten. Darin liegt ein Teil der Anpassung des justiziellen Instrumen-

36 Vgl. Kadi Barakaat/Rat und Kommission, T-315/01 und C-402/05P, Urteil vom 3. September 2008; Ayadi/Rat der Europäischen Union, T-253/02 und C-403/06, Urteil vom 3.12.2009.

37 Vgl. Beschluss des Rates vom 20. Dezember 2007 zur Änderung des Protokolls über die Satzung des Gerichtshofs, Abl. L 24, vom 29. Januar 2008, S. 42. 
tariums an das Faktum eines europäischen Kriminaljustizsystems. ${ }^{38}$ Zugleich steigt der Erledigungsdruck auf EuGH und EGMR in Strafsachen ständig und stetig an. Der Prozess gegenseitiger Anerkennung mündet in die erhöhte Nachfrage nach europäischer Justiz in ihrer Funktion sozialer Integration. Nicht nur die Haft, sondern auch die Erhebung und Verwertung von Beweisen und nicht zuletzt die Verhängung von Sanktionen im Strafrechtsraum Europa werfen notwendig Fragen nach einem kohärenten europäischen Rechtsschutz auf, der - da politisch langwierig - kurzfristig nur durch europäische Gerichte zu gewährleisten sein wird. Schließlich: der Vertrag von Lissabon sieht in Art. 86 AEUV die Einrichtung einer europäischen Staatsanwaltschaft vor. Deren Regeln - materiell und prozedural - werden das europäische Kriminaljustizsystem prägen. Von der ersten Ermittlungshandlung einer solchen Behörde an, drängt sich das Strafrecht unumgehbar justizieller europäischer Kontrolle auf. In seiner jetzigen Struktur ist der Europäische Gerichtshof auf die vertiefte Europäisierung des Strafrechts unzureichend vorbereitet. Abhilfe sieht die konsolidierte Fassung des Vertrages von Lissabon in Art. 19 EU-V und vor allem in Art. 257 AEUV vor. Am Europäischen Gerichtshof könnte eine Spezialkammer für Strafsachen eingerichtet werden. Mehr Richter mit strafrechtlicher Erfahrung und Prägung als bisher könnten das Personaltableau des Europäischen Gerichtshofs sinnvoll ergänzen.

Art. 6 Abs. 2 des Vertrages von Lissabon sieht zudem vor, dass die Europäische Union der Europäischen Menschenrechtskonvention beitritt. Damit würde die Rechtsprechung des EGMR unmittelbare Geltung für alle Rechtsakte der Europäischen Union entfalten. Das Verhältnis des Luxemburger zum Straßburger Gerichtshof wird so klärungsbedürftig. Was bislang oft noch Gegenstand eines informellen Dialogs europäischer Gerichte war, um Inkohärenz oder gar Widersprüche bei der Auslegung von Grund- und Freiheitsrechten zu vermeiden, muss nun prozedural und im Kontext einer gemeinsamen Gerichtsverfassung bewältigt werden. So sind verschiedene Denkmodelle künftiger europäischer Gerichtsorganisation - beide hohen europäischen Gerichte miteinander verknüpfend - denkbar. Der Europäische Gerichtshof für Menschenrechte würde seine Kompetenz gewiss nur dann annehmen können, wenn der Rechtsweg innerhalb des Europäischen Gerichtshofs erschöpft wäre. Ein Vorlageverfahren machte Sinn, um sich einer gemeinsamen Praxis bei der Auslegung von Menschenrechten zu vergewissern. Gleichwohl wird auch ein Rechtszug von EuGH zum EGMR vonnöten sein. Um letzteren dabei nicht in die Rolle einer Revisionsinstanz zu drängen, ist zu überlegen, ob nicht eine gemeinsame, aus Richtern des EuGH wie des EGMR bestehende Kammer am Europäischen Gerichtshof für Menschenrechte über deren Auslegung entscheidet. Diese Verdichtung justizieller Kontrolle sichert individuelle Freiheit im ausdifferenzierten europäischen Kriminaljustizsystem: der Dialog höchster europäischer Gerichte mündet in eine feste Struktur gemeinsamen europäischen Judizierens - eine neue Spur europäischen Strafrechts mit der kräftigen Tendenz, die durch staatliche Kriminalpolitik verschuldete Erosion von Grundrechten zu begrenzen und wieder umzukehren.

Letztlich: Europas Juristen müssen sich fortbilden können. Damit ist nicht nur eine tradierte Vermittlung positiven Rechtsstoffs gemeint, sondern das wechselseitige

$38 \mathrm{Vgl}$. dazu Lazowski, Towards the reform of the preliminary ruling procedure in the JHA Area, in: Braum/Weyembergh, Le contrôle juridictionnel dans l'espace pénal européen, S. $211 \mathrm{ff}$. (S. 224 ff.). 
Durchdringen von praktischer Erfahrung und Prinzipien in einem europäischen Justizdialog. Der Beitrag europäischer Justiz zur politischen Integration Europas hängt dann wesentlich davon ab, ob an die Stelle von Grundrechtserosionen, die Funktionsdefizite und Steuerungsprobleme modernen Strafrechts mitdenkend, die gelebte und als unverfügbar respektierte Charta europäischer Freiheit tritt.

Die folgenden Beiträge spiegeln die Suche nach einer europäisch organisierten Justizgewalt wider und enthalten eine Auswahl der anlässlich der Luxemburger Tagung „Criminal Justice in Europe: Challenges, Principles and Perspectives“39 gehaltenen Vorträge. Die Tagung diskutierte Rolle und Funktion der Justiz gegenüber den Herausforderungen, die ihr durch Exekutive, Legislative und grenzüberschreitende Zusammenarbeit gestellt und zugemutet werden. Peter-Alexis Albrecht formuliert klare Prinzipienpostulate an den europäischen Gesetzgeber: Gerechtigkeit und Freiheit lassen sich nur in einem auf Kernbereiche begrenzten Strafrecht und durch eine unabhängige europäische Justiz gewährleisten. Dies ist das Kontrastprogramm zu erodierenden Grundrechten in der europäischen Sicherheitsgesellschaft. Lord Justice Sir John Thomas klagt dabei Strukturen ein, die der Justiz die Wahrung ihrer inneren und äußeren Unabhängigkeit erst ermöglichen. Es liegt seiner Auffassung nach auch an einer europäischen und unabhängigen Justizgewalt Fehler europäischer Strafgesetzgebung, vor allem im Bereich gegenseitiger Anerkennung, zu korrigieren. Pedro Caeiro fragt nach den Perspektiven grenzüberschreitender Zusammenarbeit und nach einer prinzipienorientierten, rechtsstaatlich abgesicherten Auflösung von Kompetenzkonflikten europäischer Justiz. Katalin Ligeti sieht in der Rechtsprechung des Europäischen Gerichtshofs, dargestellt am Beispiel der jüngsten Mantello-Entscheidung, ${ }^{40}$ Defizite an Kohärenz und grundrechtsorientierter Weiterentwicklung des Prinzips gegenseitiger Anerkennung. John Winterdyk schließlich weist aus kriminologischer Sicht auf grundlegende Defizite von Krimialjustizsystemen weltweit hin und erinnert an das Problem, dass der Grundrechtsschutz durch eine unabhängige Justiz nicht zuletzt auch eine Frage der Verteilung finanzieller Ressourcen ist. Die Beiträge eint der Gedanke, dass die Kriminalpolitik in Europa justizieller Kontrolle und wissenschaftlicher Kritik bedarf. Zu beidem beizutragen, ist Absicht dieses Hefts.

39 Luxemburg, 22. - 23. Oktober 2010.

40 EuGH, Urteil des Gerichtshofs vom 16. November 2010, Rs. C-261/09. 